Human Friendly Mechatronics. Selected Papers of the International

Conference on Machine Automation, 25-27 September, 2000, Osaka,

Japan

Edited by : M. TAKANO, E. ARAI and T. ARAI, Elsevier Science.

\title{
Haptics: A Key to Fast Paced Interactivity
}

Vincent Hayward

Center for Intelligent Machines, McGill University, Montréal, Québec, Canada

\section{INTRODUCTION}

The word "haptics" is now well accepted. Hundreds of papers are published each year on the topic of haptic devices and interfaces. Haptics, as a technological niche, has become rich with opportunities and challenges. The field borrows from, and lends to, many subjects in science and technology. Among these, two are particularly relevant: "mechatronics" on one hand, and "robot-human interaction" on the other.

Haptic devices belong to the family of mechatronic devices because their fundamental function is to take advantage of mechanical signals to provide for communication between people and machines. It follows that haptic devices must include include transducers to convert mechanical signals to electrical signals and vice-versa used in conjunction with one or several computational or data processing systems. These transducers appeal to a variety of technologies: electromechanical devices, optoelectronics, fluids, smart materials, exploiting the possibilities that exist to build highly integrated and cost effective devices.

The popularization of haptics as an area of investigation is due to the work of such pioneers as Brooks and Iwata $[1,6]$.

\section{TAKING ADVANTAGE OF THE HAPTIC CHANNEL}

It is hard to remain insensitive to the extraordinary ability of humans to mechanically experience, and act upon their surrounding world. Part of this ability is due to the sense of touch that comprises sensations of pressure, texture, puncture, thermal properties, softness, wetness, friction-induced phenomena, adhesion, micro failures, as well as local features of objects such as edges, embossings and divets. There are also vibro-tactile sensations that refer to the perception of oscillating objects in contact with the skin. In addition, proprioceptive, or kinesthetic perception, refers to the awareness of one's body state, including position, velocity and forces supplied by the muscles. Together, proprioception and tactile sensations are fundamental to manipulation and locomotion [2]. So, why are not computers and machines in general taking better advantage of these abilities? One goal of haptics is to fill this gap.

\section{AN EXAMPLE}

Consider a familiar sheet of paper, viewed as a display device. This sheet is intended to support information encoded in the form of structured discontinuities created by lay- 
ering ink on it in order to change its reflective properties. Next, consider a computer screen with graphics capabilities. It can be programmed to display information also using structured discontinuities. Analogously, a computer mouse contains fixed mechanicallyencoded information. It is not programmable. The step that was made to move from the sheet of paper to the graphics screen is analogous to the step made to move from a computer mouse to a haptic interface. Where the graphic screen can change its optical properties under computer control, a haptic device can change its mechanical properties under computer control.

The user of a conventional mouse receives almost no information from its movements. The buttons on it, for example, are considerably richer: they respond to state change of the interface. Their mechanical detent and the small acoustical noise they produce inform the user that an event has occured. Similarly, haptic interfaces attempt to make the information flow from machine to user non zero introducing an important difference, that of being programmable or computer controlled, as in the example of moving from the sheet of paper to the graphics screen. This is illustrated in Figure 1.
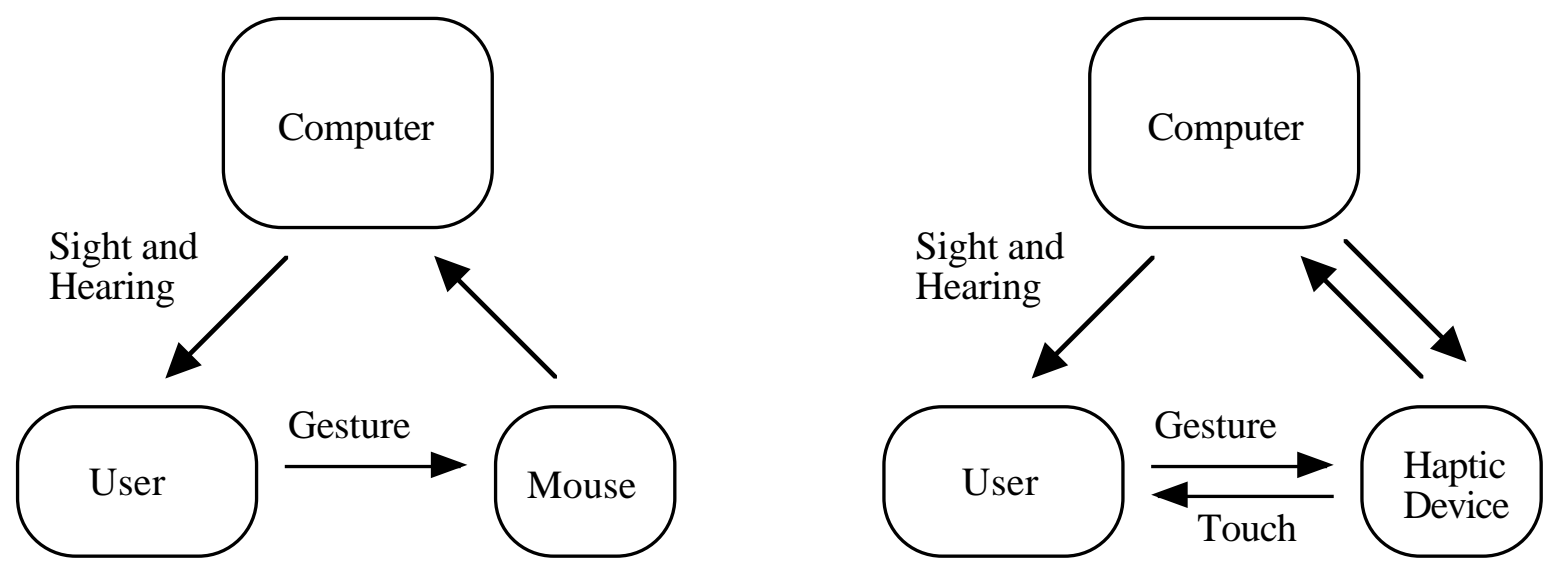

Figure 1. Left, conventional interaction loop. Right: interaction with a haptic device.

Graphical user interfaces (GUI's) have demonstrated that interactive presentation of data does not have to replicate reality, not even remotely. Being suggestive is what matters the most. Pull down menus and scrolling slider bars cannot be found elsewhere than on computer screens! The same holds for haptic interfaces. For example, the interaction forces we experience when moving objects generally occurs when they contact each other. With haptics, we can perfectly suggest a relationship between two distinct objects by creating a mutual interaction force, even if they are presented as being far apart.

Alternatively, some applications must recreate actual tasks. In other words, haptic interfaces should be designed to provide for a high fidelity reproduction of the phenomena which occur during actual manipulation. This is what in computer graphics is called the "quest for realism" and applies to haptic as well. The training of sensory-motor skills is one example of need for realism. 


\section{DEVICE EXAMPLES}

The evolution of haptic technology can be appreciated appreciated by comparing three planar haptic devices, realized over a span of six years, see Figure 2.
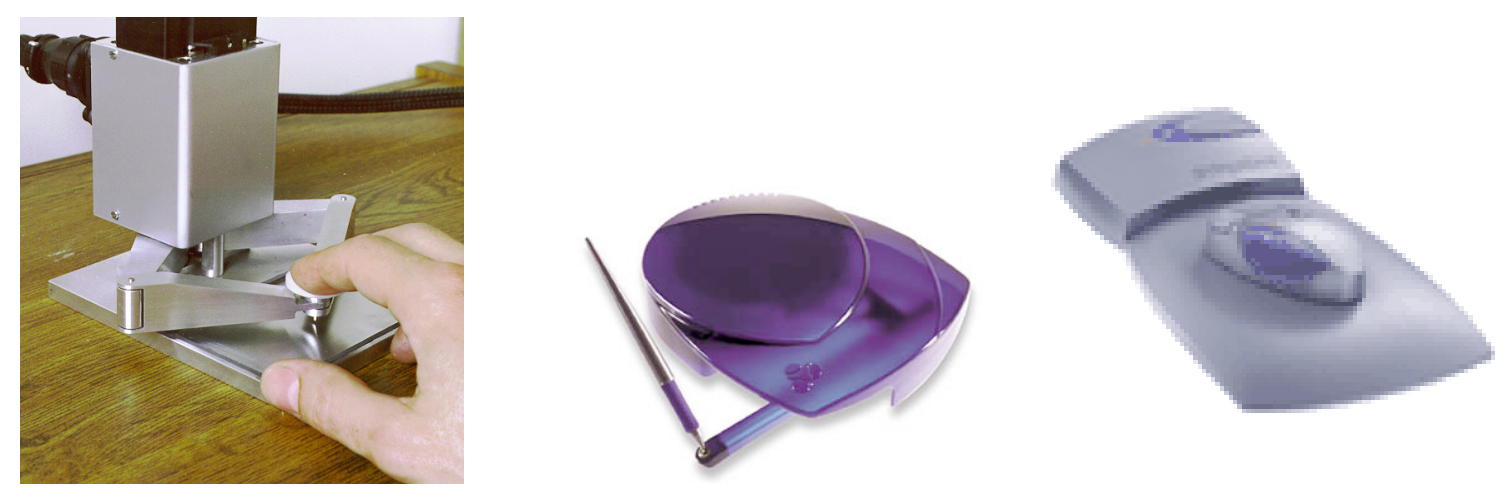

Figure 2. Left: The Pantograph [3], custom precision machining, off-the-shelf components. Middle: The PenCat/Pro ${ }^{\mathrm{TM}}$ by Immersion Canada Inc. Right: The Wingman ${ }^{\mathrm{TM}}$ by Logitech, a highly integrated device.

Other classes of devices with three or more degrees of freedom follow a similar evolution. For a recent survey see [5].

\section{APPLICATION EXAMPLES}

Bi-directionality is the single most distinguishing feature of haptic devices when compared to other machine interfaces, and this observation explains in part why they create a strong sensations of immediacy. A haptic device must be designed to "read and write" to and from the human hand (or foot, or other body parts). This combined "read and write" property may explain why the first applications of this technology involves "fastpaced interactivity" as testified by the commercial availability of haptic devices for gaming applications.

One application we have explored is the use of haptic feedback in stressful, and high paced environments, namely the operation of a GUI in absence of gravity. Haptic communication in this case could be used to reduce reliance on vision and possibly compensate for other factors caused by this type of environment [7]. Closer to everyday use, haptic devices have already been commercially introduced in certain high-end cars.

One other class of application of is interest to our group. It is the use of haptic feedback to assist operators to deal with complicated three dimensional data sets. A case presently under study is the manipulation of three dimensional angiograms which are particularly ambiguous when experienced graphically. For example, inspection of the views in Figure 3 indicate that it is difficult to realize that the shape of a vessel system could actually be highly organized. While stereopsis is helpful, the haptic experience of this system of curves should be very effective and fast [9]. 

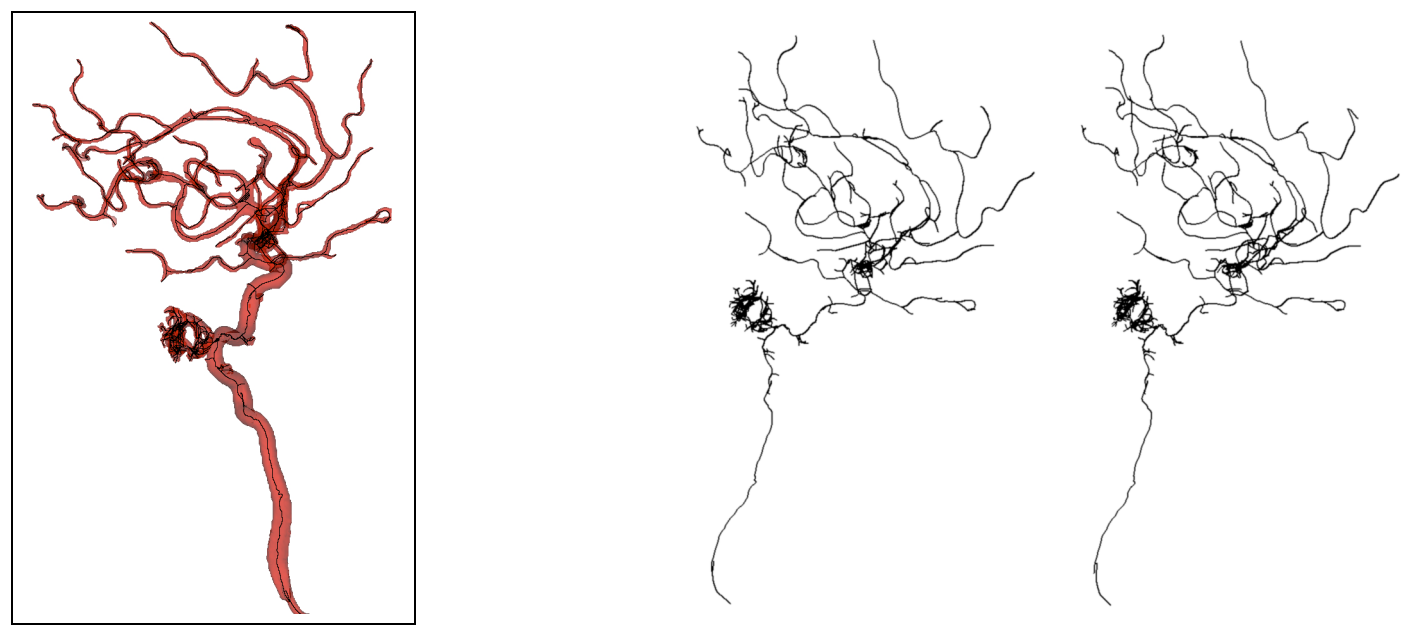

Figure 3. Left: Surface rendered vessel system. Right: a pair of stereoscopic views of its skeleton. They may be fused into a 3D image by uncrossing the eyes so that the left eye sees the left panel and the right eye sees the right panel. It is possible to realize that, despite the apparent complexity of the projection, the vessel system is actually divided into two distinct and highly organized subsystems, one in the back and one in the front.

\section{HUMAN PERCEPTION}

Our group is also exploring fascinating perceptual phenomena involving the haptic perception of shape, where contrary to intuition, force modulation as a function of movement seems to be a more determining factor of shape perception than geometrically induced limb movements [8]. In this study, it was shown that shapes could be "virtually experienced" when no geometrical information was available to the subject, see Figure 4.

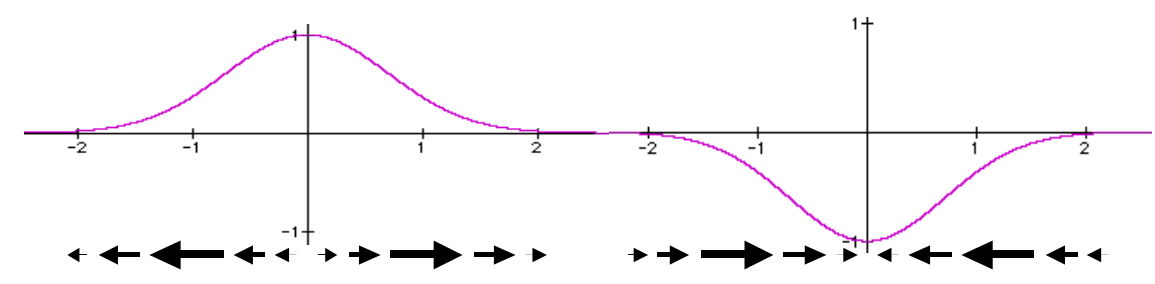

Figure 4. The arrows at the bottom show a lateral force field (LFF) that relates horizontal positions to a horizontal forces. With this stimulus, the subject receives no geometrical information, just force information, and yet what is experienced is a shape as drawn in the upper part of the figure. Note that this effect occurs over scales of centimeters and perhaps larger ones. 


\section{The Next Frontier}

Finally, "next frontier" items were discussed. One of the them is the problem of displaying tactile information directly to the skin rather than through the manipulation of a tool. In the past, tactile displays were of one of two kinds: they were either shape displays, or relied on distributed vibrotactile stimulation. A tactile display device was described which is distinguished by the fact that it relies exclusively on lateral skin stretch stimulation. It is constructed from an array of closely packed piezoelectric actuators connected to a membrane. The deformations of this membrane cause an array of contactors to create programmable lateral stress fields in the skin of the finger pad (See Figure 5)[4]. Some preliminary observations were reported with respect to the sensations that this kind of display can produce. Quite surprisingly, one of them was shape.
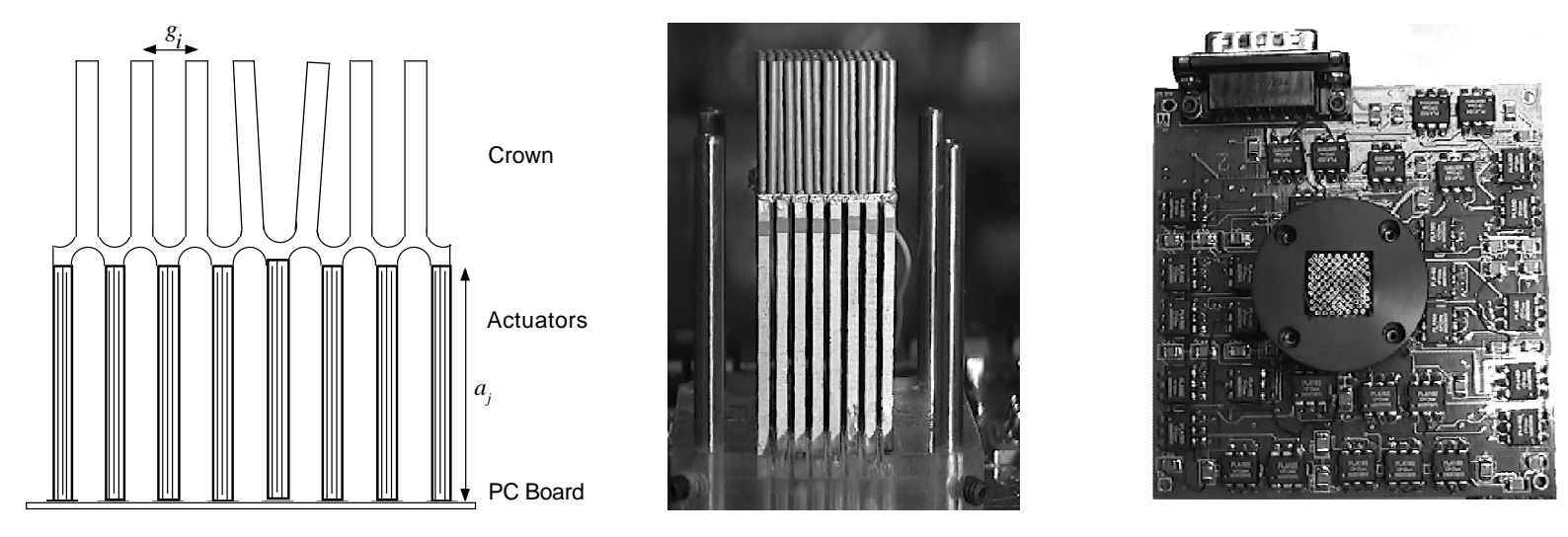

Figure 5. Left: Principle of operation. Piezoceramic actuators extend and contract, causing skin contactors to swing laterally with large mechanical movement amplification achieved through flexures. Middle: assembly detail showing and array of 64 piezoceramic actuators causing lateral swing in a array of 112 skin contactors. Right: Tactile display prototype. To give scale, the active area is $12 \times 12 \mathrm{~mm}$.

\section{CONCLUSION}

The field of haptics is developing rapidly. Haptic devices are mechatronic devices but with a special mission, that of facilitating communication between machines and humans. It is hoped that a fundamental benefit of this technology is enabling of fast paced interactivity.

Many challenges lay ahead of us to enable the progress of haptic interfaces connected with many areas of science and technology, as well as economic activity. 


\section{REFERENCES}

1. F. P. Brooks, M. Ouh-Young, J. J. Batter, and P. J. Kilpatrick. Project GROPE: Haptic displays for scientific visualization, Computer Graphics: Proc. SIGGRAPH '90, pp. 177-185, 1990.

2. J.C. Craig, G.B. Rollman. Somesthesis. Annual Review of Psychology, pp. 50:305-331 (1999).

3. V. Hayward, J. Choksi, G. Lanvin, and C. Ramstein. Design and multi-objective optimization of a linkage for a haptic interface. Advances in Robot Kinematics, pp. 352-359. J. Lenarcic and B. Ravani (Eds.), Kluver Academic (1994).

4. V. Hayward and M. Cruz-Hernandez, Tactile Display Device Using Distributed Lateral Skin Stretch, Proc. Haptic Interfaces for Virtual Environment and Teleoperator Systems Symposium, ASME, IMECE-2000. DSC-Vol. 69-2, pp. 1309-1314 (2000).

5. V. Hayward, O. R. Astley, M. Cruz-Hernandez, D. Grant, G. Robles-De-La-Torre, Haptic Interfaces and Devices. In Mechanical Systems Design (Modelling, measurement and control). CRC Press (2001). In Press.

6. H. Iwata. Artificial reality with force feedback: Development of desktop virtual space with a compact master manipulator. Computer Graphics, 24(4):165-170, (1990).

7. J. Payette, V. Hayward, C. Ramstein, D. Bergeron. Evaluation of a force-feedback (haptic) computer pointing device in zero gravity. Proc. Fifth Annual Symposium on Haptic Interfaces for Virtual Environments and Teleoperated Systems, ASME, IMECE-1996, DSC-Vol. 58. pp. 547-553 (1996).

8. G. Robles-De-La-Torre and V. Hayward, Virtual surfaces and haptic shape perception, Proc. Haptic Interfaces for Virtual Environment and Teleoperator Systems Symposium, ASME, IMECE-2000. DSC-Vol. 69-2, pp. 1081-1086 (2000).

9. D.G. Yi and V. Hayward, Skeletonization of Volumetric Angiograms for Display. In preparation. 